\title{
IDENTIFICAÇÃO DE BIÓTIPOS DE Bidens spp. RESISTENTES AOS INIBIDORES DA ALS ATRAVÉS DE Teste Germinativo ${ }^{1}$
}

\author{
Identification of Bidens spp. Biotypes Resistant to ALS-Inhibiting Herbicides Through \\ Germinative Test
}

\author{
CARVALHO, S.J.P. ${ }^{2}$, LÓPEZ-OVEJERO, R.F. ${ }^{3}$, MOYSÉS, T.C. ${ }^{2}$, CHAMMA, H.M.C.P. ${ }^{4}$ e \\ CHRISTOFFOLETI, P.J. ${ }^{5}$
}

\begin{abstract}
RESUMO - A presença de biótipos de Bidens pilosa e B. subalternans resistentes aos herbicidas inibidores da ALS nas lavouras de soja tem sido constatada em diversos locais do país. Este trabalho teve por objetivo desenvolver uma metodologia prática de detecção de biótipos resistentes de Bidens spp. aos herbicidas do grupo dos inibidores da ALS, através de testes germinativos em solução herbicida. O trabalho foi dividido em três fases de experimentos independentes. A primeira fase constituiu-se da elaboração de curvas do tipo dose-resposta para um biótipo suscetivel de Bidens spp. quando germinado em soluções de quatro herbicidas pré-emergentes (flumetsulan, diclosulan, imazaquin e metribuzin). O objetivo desta fase foi conhecer quais herbicidas se adaptam melhor à metodologia, utilizando as seguintes doses: 2C, 1C, 1/2C, 1/4C, 1/8C, 1/16C, 1/32C, 1/64C e água (em que C = dose comercial). A segunda fase constituiu-se da elaboração de curvas do tipo dose-resposta para os herbicidas selecionados na primeira fase (imazaquin e metribuzin) utilizando-se um biótipo resistente, com a intenção de identificar a dose que proporciona a maior amplitude de resposta entre os diferentes biótipos. A terceira fase constituiu-se da instalação de um teste comparativo, simultâneo, entre três biótipos resistentes e um biótipo suscetível, quando expostos à germinação nas soluções dos herbicidas selecionados na primeira fase, utilizando-se das doses escolhidas na segunda fase. Os biótipos resistentes de Bidens spp. apresentaram maior capacidade de desenvolver radículas em solução herbicida de imazaquin a $87,5 \mathrm{mg} \mathrm{L}^{-1}$ quando comparados com os biótipos suscetíveis, fato que comprova a aplicabilidade do método. A metodologia de identificação de biótipos resistentes de Bidens spp. através de testes germinativos em solução herbicida caracteriza-se como uma alternativa rápida e eficiente de identificação de biótipos.
\end{abstract}

Palavras-chave: picão-preto, germinação, resistência, identificação.

ABSTRACT - Bidens pilosa and Bidens subalternans populations resistant to ALS-inhibiting herbicides have been reported in different soybean areas of Brazil. This research aimed to develop a methodology to identify Bidens spp. biotypes resistant to ALS-inhibiting herbicides through germinative tests in herbicide solutions. The work was divided into three phases consisting of independent experiments. The first phase consisted of dose-response curves prepared for a susceptible biotype of Bidens spp., germinated in solutions of four pre-emergence herbicides (flumetsulan, diclosulan, imazaquin and metribuzin). The objective of this phase was to select the herbicides best adapted to the methodology, by using the following rates: $2 C, 1 C, 1 / 2 C, 1 / 4 C$, $1 / 8 C, 1 / 16 C, 1 / 32 C, 1 / 64 C$ and water (where $C=$ recommended rate). The second phase consisted of dose-response curves prepared for the herbicides selected in the first phase (imazaquin and metribuzin) using a resistant biotype, to identify the dose providing the largest response range between the biotypes. The third phase consisted of a comparative test between three resistant and one susceptible biotypes, exposed to germination in the solutions of the herbicides chosen in the

Recebido para publicação em 12.03.2004 e na forma revisada em 10.9.2004.

2 Aluno de graduação da Escola Superior de Agricultura "Luiz de Queiroz” - ESALQ/USP, Caixa Postal 09, 13419-900 PiracicabaSP ${ }^{3}$ Doutorando do Departamento de Produção Vegetal - ESALQ/USP; ${ }^{4}$ Técnico de Nível Superior do Laboratório de Produção de Sementes - ESALQ/USP; ${ }^{5}$ Professor Associado do Departamento de Produção Vegetal - ESALQ/USP.

Planta Daninha, Viçosa-MG, v. 22, n. 3, p. 411-417, 2004 
first phase, applying the doses chosen in the second phase. The resistant Bidens spp. biotypes demonstrated a better capacity to develop roots in imazaquin herbicide solution (87 $\mathrm{mg} \mathrm{L}^{-1}$ ), than the susceptible biotype, a fact that proves the method's applicability. The methodology applied to identify Bidens spp. resistant biotypes through germinative tests in herbicide solution is a fast and efficient alternative for biotype identification.

Key words: Bidens, germination, resistance, detection.

\section{INTRODUÇÃO}

Um dos problemas que mais preocupam atualmente a agricultura brasileira é a resistência das plantas daninhas aos herbicidas. Os casos mais freqüentes de resistência de plantas daninhas que têm surgido no Brasil estão relacionados aos herbicidas inibidores da ALS e ACCase.

Especificamente para os herbicidas inibidores da ALS foram relatados até o momento casos com as plantas daninhas Bidens pilosa, Bidens subalternans, Euphorbia heterophylla, Sagittaria montevidensis, Fimbristylis miliacea e Cyperus difformis, sendo as três primeiras na cultura da soja e as três últimas na cultura do arroz (HRAC-BR, 2004).

A extensão de áreas agrícolas com presença de biótipos resistentes de Bidens spp. aumenta em uma taxa elevada. Nas lavouras de soja das regiões Centro-Oeste e Sul, tornam-se cada vez mais freqüentes relatos de aparecimento de biótipos resistentes de Bidens spp. aos inibidores da ALS. Essa planta daninha, pertencente à família Asteraceae, tem sua origem na América tropical, com maior presença na América do Sul. Possui grande dispersão mundial e está presente, também, em quase todo o território nacional, principalmente nas áreas agrícolas da região Centro-Sul (Kissmann \& Groth, 1999).

A resistência de plantas daninhas aos herbicidas é a capacidade natural e herdável de alguns biótipos, dentro de uma determinada população, de sobreviver e se reproduzir após a exposição à dose de um herbicida que seria letal a uma população normal (suscetivel) da mesma espécie (Christoffoleti \& LópezOvejero, 2003).

Além de provocar perdas de produtividade devido à interferência sobre as plantas cultivadas, a problemática causada pela presença do picão-preto é agravada, uma vez que já ocorreram diversos casos comprovados de resistência do complexo Bidens pilosa-Bidens subalternans aos herbicidas inibidores da enzima acetolactato sintase (ALS) no Brasil (Vidal \& Fleck, 1997; Ponchio, 1997). O uso prolongado de um mesmo herbicida, ou o uso repetitivo de químicos que possuem o mesmo mecanismo de ação em culturas sucessivas, eleva o potencial de surgimento de casos de resistência na área (Vidal \& Merotto Jr., 1999).

Gelmini (2001) comprovou a existência de biótipos de picão-preto com resistência cruzada aos inibidores da ALS dos grupos das sulfoniluréias e imidazolinonas. A resistência das plantas daninhas aos herbicidas inibidores da ALS é o resultado de uma alteração do gene responsável pela codificação desta enzima (Shaner, 1991). A pressão de seleção gerada pelos herbicidas, por sua vez, aumenta a freqüência dos biótipos resistentes (Rizzardi et al., 2002).

A partir desses fatos, surgiu a necessidade de metodologias rápidas e precisas, capazes de detectar biótipos resistentes de plantas daninhas. Ryan (1970), visando a comprovação da existência de biótipos resistentes de plantas daninhas, desenvolveu um protocolo de estudo científico em casa de vegetação.

Christoffoleti (2002) propõe a caracterização do nível de resistência de biótipos de plantas daninhas aos herbicidas inibidores da ALS através da construção de curvas dose-resposta. Oliveira et al. (2002) estudaram o efeito do herbicida imazaquin na atividade in vitro da ALS sobre genótipos de milho e biótipos resistentes e suscetíveis de amendoim-bravo (Euphorbia heterophylla), concluindo ser este um método sensivel para detecção de biótipos resistentes.

Com relação à germinação, Garcia (1987) afirma ser o intervalo de temperatura de $20 / 30{ }^{\circ} \mathrm{C}$ ( 8 horas de luz e 16 horas de escuro) 
aquele que proporciona elevada taxa de germinação das sementes de picão-preto. Segundo Reddy \& Singh (1992), o picão-preto possui temperatura ótima dentro do intervalo de $25 / 20$ a $35 / 30{ }^{\circ} \mathrm{C}$ (dia/noite, $12 / 12 \mathrm{~h}$ ).

Adegas et al. (2003) afirmam que, em todos os períodos de embebição em que as sementes foram expostas, a germinação iniciou-se no terceiro dia e encerrou-se no sétimo e que, à medida que se aumentou o período de embebição, ocorreu o acréscimo da taxa inicial de germinação.

Dessa forma, o objetivo deste trabalho foi desenvolver uma metodologia prática de identificação de biótipos resistentes de Bidens spp. aos herbicidas do grupo dos inibidores da ALS através de testes germinativos em solução herbicida.

\section{MATERIAL E MÉTODOS}

O experimento foi conduzido no Laboratório de Produção de Sementes da Escola Superior de Agricultura "Luiz de Queiroz", Piracicaba-SP, durante os meses de agosto a dezembro de 2003; cada teste teve duração de sete dias. Com base na metodologia de Garcia (1987), durante todo o trabalho, os testes germinativos foram instalados em câmara de germinação com controle constante de temperatura e umidade ( 16 horas de escuro a $20^{\circ} \mathrm{C}$ e 8 horas de luz a $30^{\circ} \mathrm{C}$ ).

O delineamento experimental adotado foi de blocos ao acaso, com quatro repetições. Cada bloco localizou-se em uma prateleira da câmara de germinação. As parcelas constituíram-se de caixas plásticas transparentes
(11 x $11 \times 3 \mathrm{~cm})$, contendo 50 sementes de Bidens spp., e duas folhas de papel mata-borrão (10,5 x 10,5 cm); o volume de solução aplicado correspondeu a duas vezes e meia o peso total do papel seco.

O trabalho foi dividido em três fases de experimentos, analisados de forma independente. Na primeira e segunda fases realizouse a contagem do número de sementes germinadas aos 3, 5 e 7 dias após instalação (DAI), bem como do número de plântulas normais, anormais tipo I (parte aérea normal, porém com radícula menor que $1,5 \mathrm{~cm}$ ), anormais tipo II (plântulas completamente anormais) e sementes não-germinadas aos 7 DAI, ajustados à porcentagem. Na terceira fase realizou-se, também, a medição da radícula de 10 plântulas com parte aérea, ao acaso, por parcela, aos 7 DAI. Considerou-se germinada toda semente que apresentasse comprimento de radícula superior a 2,0 $\mathrm{mm}$ (Voll et al., 2003; Azania et al., 2003).

A primeira fase constituiu-se da elaboração de curvas do tipo dose-resposta para um biótipo suscetível de Bidens, sobre a variável plântulas anormais tipo II, quando germinado em soluções de flumetsulan, diclosulan, imazaquin (inibidores de ALS) e metribuzin (inibidor do fotossistema II). Os tratamentos encontram-se na Tabela 1 . O objetivo da primeira fase foi selecionar herbicidas que se adaptassem à metodologia: um herbicida do grupo dos inibidores da ALS e um herbicida "testemunha" de outro mecanismo de ação. O critério para a seleção do herbicida foi alcançar, no mínimo, o valor de 90\% de plântulas anormais tipo II para as doses mais concentradas da curva.

Tabela 1 - Soluções de herbicidas utilizadas na elaboração das curvas de dose-resposta

\begin{tabular}{|l|c|c|c|c|c|c|c|c|c|}
\hline \multicolumn{1}{|c|}{ Herbicida } & \multicolumn{10}{|c|}{ Solução em mg L } \\
\hline \multicolumn{1}{|c|}{$i . a}$. & $\mathrm{T}$ & $1 / 64 \mathrm{C}$ & $1 / 32 \mathrm{C}$ & $1 / 16 \mathrm{C}$ & $1 / 8 \mathrm{C}$ & $1 / 4 \mathrm{C}$ & $1 / 2 \mathrm{C}$ & $\mathrm{C}^{*}$ & $2 \mathrm{C}$ \\
\hline Diclosulan & 0,00 & 2,62 & 5,25 & 10,50 & 21,00 & 42,00 & 84,00 & 168,00 & 336,00 \\
\hline Flumetsulan & 0,00 & 9,38 & 18,75 & 37,50 & 75,00 & 150,00 & 300,00 & 600,00 & $1.200,00$ \\
\hline Imazaquin & 0,00 & 10,94 & 21,88 & 43,75 & 87,50 & 175,00 & 350,00 & 700,00 & $1.400,00$ \\
\hline Metribuzin** & 0,00 & 37,50 & 75,00 & 150,00 & 300,00 & 600,00 & $1.200,00$ & $2.400,00$ & $4.800,00$ \\
\hline
\end{tabular}

* Solução oriunda da dissolução da dose comercial em volume equivalente a 200 L; ** Herbicida "testemunha" de outro mecanismo de ação; i.a.: ingrediente ativo. 
A segunda fase constituiu-se da elaboração de curvas do tipo dose-resposta, sobre a variável plântulas anormais tipo II, para um biótipo com resistência comprovada em casa de vegetação, utilizando-se os herbicidas selecionados na primeira fase. $\mathrm{O}$ objetivo da segunda fase foi comparar as curvas definidas na primeira fase (biótipo suscetivel) com as curvas elaboradas para o biótipo resistente e, assim, identificar a dose que promoveria a maior amplitude de resposta da variável plântulas anormais tipo II entre os biótipos, quando germinados nas soluções herbicidas. Os tratamentos utilizados foram os mesmos da primeira fase.

Para elaboração das curvas de dose-resposta da primeira e segunda fases, os dados de plântulas anormais tipo II foram ajustados a um modelo de regressão não-linear, por meio do programa estatístico TableCurve, usando a equação logística dose-resposta:

$$
y=a+\frac{b}{\left[1+\left(\frac{x}{c}\right)^{d}\right]}
$$

em que $y$ é a porcentagem de plântulas anormais tipo II, $x$ é a concentração da solução em mg i.a. $\mathrm{L}^{-1}$ e $a, b, c$ e $d$ são coeficientes da curva, de modo que $a$ é o limite inferior da curva, $b$ é a diferença entre o ponto máximo e o mínimo da curva, $c$ é a dose que proporciona $50 \%$ de plântulas anormais tipo II e $d$ é a declividade da curva (Seefeldt et al., 1995).

A terceira fase constituiu-se de um teste comparativo, simultâneo, entre três biótipos comprovadamente resistentes e um biótipo suscetível de Bidens spp., quando expostos à germinação na solução dos herbicidas selecionados na primeira fase, com as doses escolhidas na segunda fase. Os tratamentos foram arranjados em esquema fatorial com os quatro biótipos de Bidens e três condições de germinação: solução de um herbicida inibidor da ALS, solução de um herbicida inibidor do fotossistema II e somente água, totalizando 12 tratamentos.

Os dados de plântulas normais e anormais tipo II, bem como os de comprimento radicular, para os quatro biótipos, obtidos na terceira fase, foram avaliados segundo o teste $\mathrm{F}$ aplicado sobre a análise da variância, seguido do teste de Tukey $(\alpha=0,01)$.

\section{RESULTADOS E DISCUSSÃO}

Em relação à primeira fase, obteve-se a definição das curvas do tipo dose-resposta de Bidens spp. para os herbicidas flumetsulan, diclosulan, imazaquin e metribuzin. Com base na variável plantas anormais tipo II, realizouse o descarte dos herbicidas flumetsulan e diclosulan, uma vez que estes não alcançaram o valor mínimo crítico de $90 \%$ de plântulas anormais tipo II para as doses mais concentradas da curva, não se adaptando à metodologia proposta (Figura 1).

Assim, como resultado da primeira fase, obteve-se a seleção do herbicida imazaquin como representante do grupo dos inibidores da ALS e a seleção do metribuzin como herbicida "testemunha" de outro mecanismo de ação (inibidor do fotossistema II) (Figura 2).

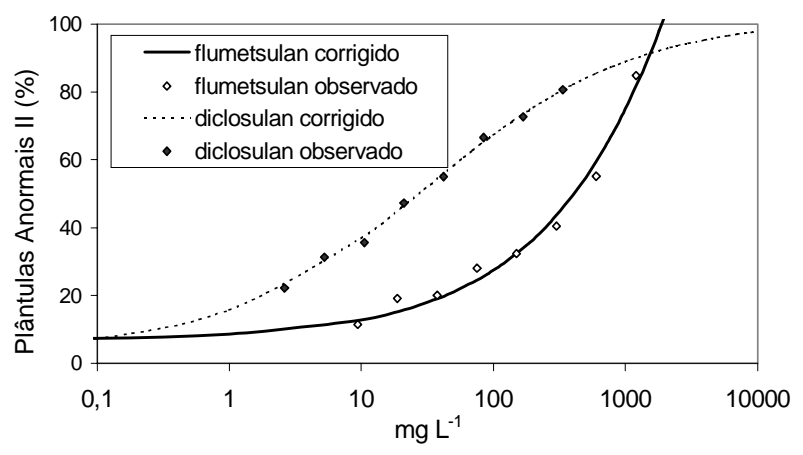

Figura 1 - Plantas anormais tipo II (\%) do biótipo suscetível quando germinado em solução de flumetsulan e diclosulan.

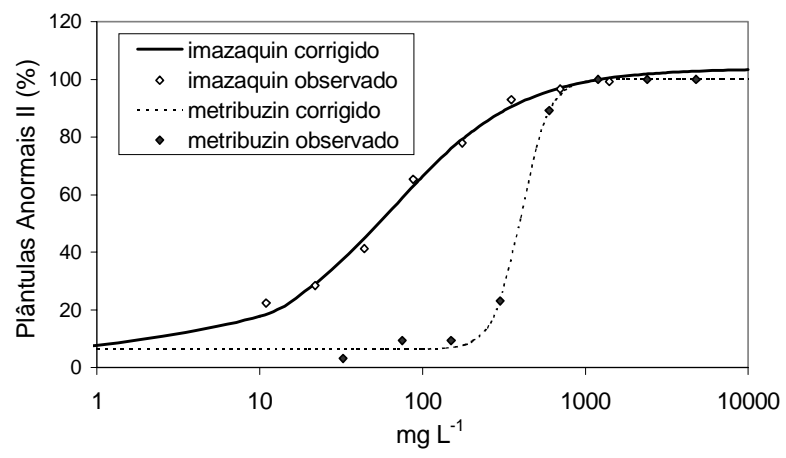

Figura 2 - Plantas anormais tipo II (\%) do biótipo suscetível quando germinado em solução de imazaquin e metribuzin. 
Na segunda fase, obteve-se a definição das curvas, dose-resposta para os herbicidas imazaquin e metribuzin, considerando-se o biótipo resistente. Para todas as curvas do trabalho, os parâmetros $a, b, c$ e $d$ da equação logística dose-resposta encontram-se na Tabela 2.

A partir da sobreposição das curvas do herbicida imazaquin geradas pela primeira e segunda fases (Figura 3), pôde-se prever que a dose que promove a maior amplitude de resposta para a variável avaliada (plântulas anormais tipo II) localiza-se próxima da concentração de $87,5 \mathrm{mg} \mathrm{L}^{-1}(1 / 8 \mathrm{C})$.

O herbicida metribuzin, quando em concentrações superiores a $300 \mathrm{mg} \mathrm{L}^{-1}$, agiu como inibidor da emissão de radículas (germinação), alcançando valores próximos de $100 \%$ de sementes mortas na dose mais concentrada da curva. Somente para a elaboração do gráfico, considerou-se $100 \%$ de sementes mortas equivalente a $100 \%$ de plântulas anormais tipo II (Figura 4). O herbicida imazaquin não agiu como inibidor da germinação em nenhuma das doses.

A terceira fase mostrou o comportamento dos três biótipos resistentes e do biótipo suscetivel quando expostos às mesmas condições de germinação, simultaneamente. Na análise dos dados obtidos pela variável plântulas normais, percebeu-se uma tendência de os biótipos resistentes apresentarem maiores porcentagens de plântulas normais do que o biótipo suscetível, quando germinados em solução de imazaquin a $87,5 \mathrm{mg} \mathrm{L}^{-1}$. No entanto, a análise estatística dos dados através do teste $\mathrm{F}$ aplicado sobre a análise da variância, seguido do teste de Tukey $(\alpha=0,01)$, não mostrou diferença entre os biótipos.
Da mesma forma, os biótipos resistentes apresentaram menores valores percentuais de plântulas anormais tipo II quando comparados com o biótipo suscetível; contudo, a análise estatística dos dados através do teste $\mathrm{F}$ aplicado sobre a análise da variância, seguido do teste de Tukey $(\alpha=0,01)$, também não apresentou diferença entre os biótipos, devido à elevada variação entre repetições.

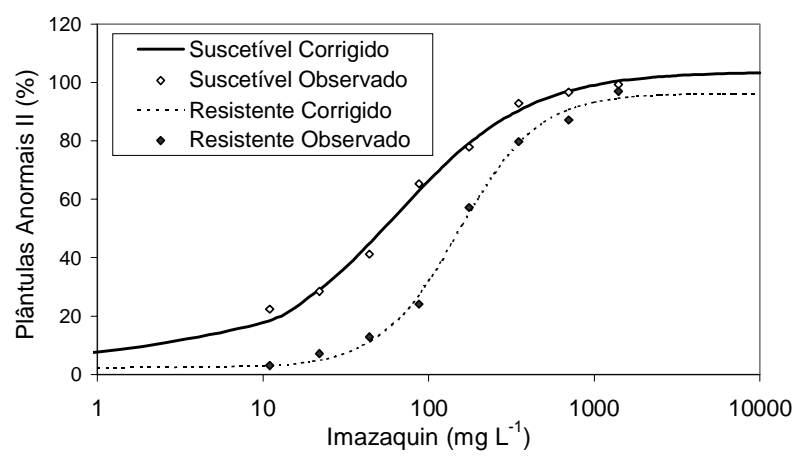

Figura 3 - Plantas anormais tipo II (\%) dos biótipos suscetível e resistente quando germinados em solução de imazaquin.

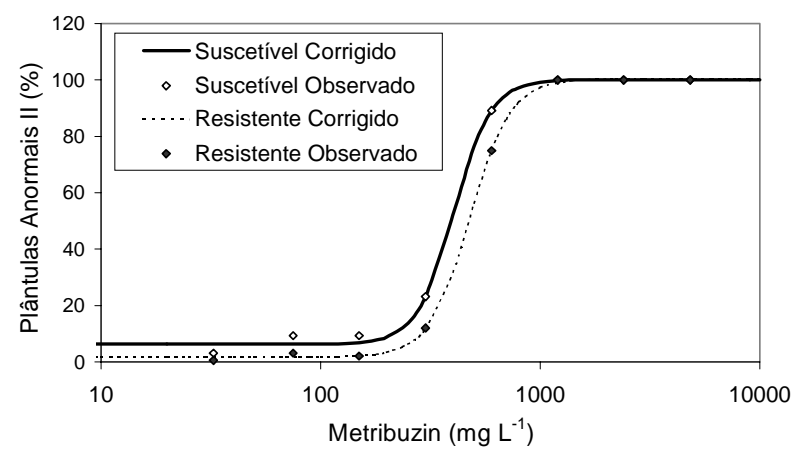

Figura 4 - Plantas anormais II (\%) dos biótipos suscetível e resistente quando germinados em solução de metribuzin.

Tabela 2 - Parâmetros $a, b, c$ e $d$ da equação logística dose-resposta dos biótipos resistente e suscetível, para a variável plântulas anormais tipo II, com os herbicidas testados

\begin{tabular}{|l|c|c|c|c|c|c|c|c|}
\hline \multirow{2}{*}{ Herbicida } & \multicolumn{4}{|c|}{ Biótipo Suscetível } & \multicolumn{4}{c|}{ Biótipo Resistente } \\
\cline { 2 - 9 } & $a$ & $b$ & $c$ & $d$ & $a$ & $b$ & $c$ & $d$ \\
\hline Flumetsulan & 6,8653 & 806,754 & 77557,2 & $-0,5476$ & -- & -- & -- & -- \\
\hline Diclosulan & 3,6210 & 98,0186 & 33,0167 & $-0,5589$ & -- & -- & -- & -- \\
\hline Imazaquin & 6,7226 & 96,9833 & 65,2663 & $-1,0978$ & 2,2108 & 94,0579 & 152,312 & $-1,8092$ \\
\hline Metribuzin & 6,2718 & 93,8916 & 403,712 & $-5,0687$ & 1,6824 & 98,7541 & 477,689 & $-4,6494$ \\
\hline
\end{tabular}


Na análise dos dados obtidos pela variável "média do comprimento radicular" de 10 plantas com parte aérea, percebeu-se a tendência de os biótipos resistentes apresentarem-se mais capazes de desenvolver radículas que o biótipo suscetível, quando germinados em solução de imazaquin a $87,5 \mathrm{mg} \mathrm{L}^{-1}$. A análise estatística dos dados por meio do teste $\mathrm{F}$ aplicado sobre a análise da variância, seguido do teste de Tukey $(\alpha=0,01)$, mostrou que a variável satisfaz a necessidade da metodologia em desenvolvimento, uma vez que os biótipos resistentes e o biótipo suscetivel comportaram-se de forma diferente para essa variável (Tabela 3).

Tabela 3 - Comprimento médio radicular $(\mathrm{cm})$ dos quatro biótipos de picão-preto quando expostos a diferentes condições de germinação

\begin{tabular}{|l|c|c|c|}
\hline \multirow{2}{*}{ Biótipo } & \multicolumn{4}{|c|}{ Solução } \\
\cline { 2 - 4 } & água & imazaquin & metribuzin \\
\hline Suscetível & $2,355 \mathrm{~A} \mathrm{a}$ & $0,820 \mathrm{~B} \mathrm{~b}$ & $1,370 \mathrm{~B}$ a \\
\hline Resistente 1 & $1,960 \mathrm{~A} \mathrm{a}$ & $1,923 \mathrm{~A} \mathrm{a}$ & $1,097 \mathrm{~B}$ a \\
\hline Resistente 2 & $2,700 \mathrm{~A} \mathrm{a}$ & $1,835 \mathrm{~B}$ a & $1,575 \mathrm{~B}$ a \\
\hline Resistente 3 & $2,450 \mathrm{~A}$ a & $1,75 \mathrm{AB}$ a & $1,730 \mathrm{~B}$ a \\
\hline
\end{tabular}

*Valores seguidos por letras maiúsculas iguais na linha $(\mathrm{DMS}=0,701)$ ou letras minúsculas iguais na coluna (DMS $=0,754)$ não diferem segundo o teste de Tukey ( $\alpha=0,01$ ).

De forma geral, os biótipos resistentes e o biótipo suscetivel comportaram-se de maneira semelhante quando germinados em solução de metribuzin a $300 \mathrm{mg} \mathrm{L}^{-1}$ e em água, para todas as variáveis.

Assim, após a execução do presente trabalho, pôde-se concluir que: o herbicida imazaquin presta-se à metodologia de detecção de biótipos resistentes de Bidens spp. através de testes germinativos em solução herbicida; a dose discriminatória do imazaquin que promove a maior amplitude de resposta entre o biótipo suscetível e o biótipo resistente, para a variável plântulas anormais tipo II, localizase próxima da concentração de $87,5 \mathrm{mg} \mathrm{L}^{-1}$; o herbicida metribuzin é inibidor da germinação quando em doses superiores a $300 \mathrm{mg} \mathrm{L}^{-1} \mathrm{e}$ não age de forma distinta sobre qualquer biótipo testado; a variável comprimento médio radicular de plântulas com parte aérea mostrou-se bastante adequada à metodologia em questão; e a metodologia de detecção de biótipos resistentes de Bidens spp. através de testes germinativos em solução herbicida caracterizou-se como uma alternativa rápida (7 dias) e eficiente de identificação de biótipos.

\section{AGRADECIMENTOS}

Ao Laboratório de Produção de Sementes da Escola Superior de Agricultura "Luiz de Queiroz", por todo o apoio recebido.

\section{LITERATURA CITADA}

ADEGAS, F. S.; VOLL, E.; PRETE, C. E. C. Embebição e germinação de sementes de picão-preto (Bidens pilosa). Planta Daninha, v. 21, n. 1, p. 21-25, 2003.

AZANIA, A. A. P. M. et al. Métodos de superação de dormência em sementes de Ipomoea e Merremia. Planta Daninha, v. 21, n. 2, p. 203-209, 2003.

CHRISTOFFOLETI, P. J. Curvas de dose-resposta de biótipos resistente e suscetível de Bidens pilosa L. aos herbicidas inibidores da ALS. Sci. Agric., v. 59, n. 3, p. 513519, 2002.

CHRISTOFFOLETI, P. J.; LÓPEZ-OVEJERO, R. F. Definições e situação da resistência de plantas daninhas aos herbicidas no Brasil e no mundo. In: CHRISTOFFOLETI, P. J. Aspectos de resistência de plantas daninhas a herbicidas. Londrina: Associação Brasileira de Ação a Resistência de Plantas aos Herbicidas, 2003. p. 2-21. (HRAC-BR)

GARCIA, A. R. Estudo de fatores do ambiente na germinação de frutos polimórficos de Bidens pilosa $\mathrm{L}$. 1987. 60 f. Dissertação (Mestrado em Ciência Florestal) Universidade Federal de Viçosa, Viçosa, 1987.

GELMINI, G. A. Resistência de biótipos de Euphorbia heterophylla L., Bidens subalternans L. e Brachiaria plantaginea (Link) Hitchc. a herbicidas utilizados na cultura da soja (Glycine max (L.) Merril). 2001. $147 \mathrm{f}$. Dissertação (Mestrado em Fitotecnia) - Escola Superior de Agricultura “Luiz de Queiroz", Piracicaba, 2001. 147 p.

ASSOCIAÇÃO BRASILEIRA DE AÇÃO A RESISTÊNCIA DE PLANTAS AOS HERBICIDAS - HRAC-BR.

Disponible em: <www.hrac-br.com.br/>. Acesso em: 2004.

KISSMANN, C. G.; GROTH, D. Plantas infestantes e nocivas. São Paulo: BASF, 1999. 978 p. t. 2.

OLIVEIRA, M. F. et al. Atividade da acetolactato sintase de plantas de milho e de amendoim-bravo (Euphorbia heterophylla) resistentes e suscetíveis ao imazaquin. Planta Daninha, v. 20, n. 1, p. 77-82, 2002. 
PONCHIO, J. A. R. Resistência de biótipos de Bidens pilosa L. a herbicidas inibidores da enzima ALS/AHAS. 1997. 120 f. Tese (Doutorado em Fitotecnia) - Escola Superior de Agricultura “Luiz de Queiroz", Piracicaba, 1997.

REDDY, K. N.; SINGH, M. Germination and emergence of hairy beggarticks (Bidens pilosa). Weed Sci., v. 40, n. 2, p. 195-199, 1992.

RIZZARDI, M. A. et al. Resistência de plantas aos herbicidas inibidores da acetolactato sintase. Planta Daninha, v. 20, n. 1, p. 149-158, 2002.

RYAN, G. F. Resistance of commom groundsel to simazine and atrazine. Weed Sci., v. 18, p. 614-620, 1970.

SEEFELDT, S. S.; JENSEN, S. E.; FUERST, E. P. Loglogistic analysis of herbicide dose-response relationship. Weed Technol., v. 9, p. 218-227, 1995.
SHANER, D. L. Mechanisms of resistance to acetolactate synthase/acetohydroxyacid synthase inhibitors. In: CASELEY, J. C.; CUSSANS, G. W.; ATKIN, R. K. Herbicide resistance in weeds and crops. Oxford: Butterworth-Heinemann, 1991. p. 27-43.

VIDAL, R. A.; FLECK, N. G. Three weed species with confirmed resistance to herbicides in Brazil. In: MEETING OF THE WEED SCIENCE SOCIETY OF AMERICA, 37., 1997, Orlando. Abstract... Orlando: Weed Science Society of America, 1997. p. 100.

VIDAL, R. A.; MEROTTO Jr., A. Resistência de amendoim-bravo aos herbicidas inibidores da enzima acetolactato sintase. Planta Daninha, v. 17, n. 3, p. 367373, 1999.

VOLL, E. et al. Relações entre germinação de sementes de espécies de plantas daninhas e uso da condutividade elétrica. Planta Daninha, v. 21, n. 2, p. 181-189, 2003. 